Proyecciones

Vol. 19, No 2, pp. 141-155, August 2000

Universidad Católica del Norte

Antofagasta - Chile

\title{
PRINCIPAL NORMAL SPECTRAL VARIATIONS OF SPACE CURVES
}

\author{
M. PETROVIĆ, J. VERSTRAELEN \\ and L. VERSTRAELEN \\ University of Kragujevac - Yugoeslavia, \\ Katholieke Universiteit Brussel - Belgium, \\ Katholieke Universiteit Leuven - Belgium
}

\begin{abstract}
In the present paper, we give a similar spectral variational theory for closed curves in the Euclidean 3-space $E^{3}$, considering deformations in the direction of the principal normal vector field. Similarly as in the planar case, the closed Euclidean space curves satisfying the corresponding variational minimal principle are characterized by their curvature being a function of finite Chen type; their torsion remains completely free.
\end{abstract}




\section{Introduction}

In [6], B.Y.Chen, F.Dillen, L.Vrancken and one of the authors introduced the notion of $k$-minimality for compact hypersurfaces in Riemannian manifolds, whereby $k \in N$, and, in particular, 0-minimality corresponds to the classical notion of minimality (i.e. vanishing mean curvature function), and 1-minimality corresponds to CMC (i.e. $H$ is constant). Basically, this is done by imposing these hypersurfaces to satisfy a variational minimal principle for their volume (area when dimension is 2 , length when dimension is 1) under so-called normal $k$-deformations, i.e. normal deformations for which the variational functions are chosen in relation with elementary spectral theory. In particular, in [] the closed $k$-minimal curves in the Euclidean plane $E^{2}$ are characterized by the property that their curvature function is of finite type, i.e. has a finite Fourier series expansion with respect to the arclength. As such, all 2-minimal closed Euclidean planar curves were classified, and also many examples of such $k$-minimal curves, for $k>2$, were given.

In the present paper, we give a similar spectral variational theory for closed curves in the Euclidean 3-space $E^{3}$, considering deformations in the direction of the principal normal vector field. Similarly as in the planar case, the closed Euclidean space curves satisfying the corresponding variational minimal principle are characterized by their curvature being a function of finite Chen type; their torsion remains completely free. Finally, we discuss some examples of closed k-minimal space curves, which were studied before in the context of the theory of curves of finite Chen type [3], [4], [7], [8], [9] and which came up very recently in the work of P.D.Scofield on space curves of constant precession [10].

\section{Deformations of space curves in the principal normal direction}

Let $\beta$ be a closed (regular) curve in $E^{3}$ of length $2 \pi r, r \in R_{0}^{+}$. Then $\beta$ can be considered as an isometric immersion of a circle $S^{1}(r)$ of radius $r$ into $E^{3}$ :

$$
\beta: S^{1}(r) \rightarrow E^{3} s \mapsto \beta(s),
$$


whereby $s \in[0,2 \pi r]$ is an arclength parameter. As usual, let $T=\beta^{\prime}$, $N$ and $B$ be the Frenet trihedral of $\beta$, such that the curvature function $k(s)$ and the torsion function $\tau(s)$ of $\beta$ are determined by the Frenet formulas $T^{\prime}=k N, N^{\prime}=-k T+\tau B$ and $B^{\prime}=-\tau N$.

Now, we consider deformations of the curve $\beta$ in the direction of its principal normal $N$, i.e. variations given by

$$
\beta \rightarrow \beta_{t} s \mapsto \beta_{t}(s):=\beta(s)+t f(s) N(s),
$$

where $t \in(-\epsilon, \epsilon), \epsilon \in R_{0}^{+}$, and $f \in C^{\infty} \beta$; of course, $\beta=\beta_{0}$.

From (2.2) it follows that the velocity of the deformed curve $\beta_{t}$ is given by

$$
\beta_{t}^{\prime}=\{1-t k f\} T+t f^{\prime} N+t \tau f B,
$$

(when' denotes differentiation with respect to $s$ ). Hence,

$$
\left\|\beta_{t}^{\prime}\right\|^{2}=1-(2 k f) t+\left(k^{2} f^{2}+\tau^{2} f^{2}+f^{\prime 2}\right) t^{2} .
$$

Consequently, the length $L(t)$ of the curve $\beta_{t}$ is found to be

$$
\begin{array}{r}
L(t)=\int_{0}^{2 \pi \tau}\left\|\beta_{t}^{\prime}(s)\right\| s=\int_{0}^{2 \pi \tau}(1-(2 k f) t+ \\
\left.=\left(k^{2} f^{2}+\tau^{2} f^{2}+f^{\prime 2}\right) t^{2}\right)^{\frac{1}{2}} d s \\
=\int_{0}^{2 \pi \tau}\left(1-A t+B t^{2}\right)^{\frac{1}{2}} d s
\end{array}
$$

where we have put

$$
A=2 k f \quad, \quad B=k^{2} f^{2}+\tau^{2} f^{2}+f^{\prime 2} .
$$

Thus we find the following first variational formula for the length under these deformations:

$$
L^{\prime}(t)=\int_{0}^{2 \pi r} \frac{1}{2} \frac{-A+2 B t}{\left(1-A t+B t^{2}\right)^{\frac{1}{2}}} d s .
$$

This implies that

$$
L^{\prime}(0)=-\frac{1}{2} \int_{0}^{2 \pi r} A d s=-\int_{0}^{2 \pi r} k(s) f(s) d s .
$$

Moreover, from (2.7), we obtain the following second variational formula:

$$
L^{\prime \prime}(t)=\int_{0}^{2 \pi r} \frac{1}{4} \frac{4 B\left(1-A t+B t^{2}\right)-(-A+2 B t)^{2}}{\left(1-A t+B t^{2}\right)^{\frac{3}{2}}} d s .
$$


This implies that

$$
L^{\prime \prime}(0)=\int_{0}^{2 \pi r} \frac{1}{4}\left(4 B-A^{2}\right) d s=\int_{0}^{2 \pi r}\left(\tau^{2}(s) f^{2}(s)+f^{\prime 2}(s)\right) d s .
$$

Definition. Let $D^{N}$ be the set of all deformations of a space curve $\beta$ in the direction of its principal normal $N$. Then $\beta$ is a minimal curve when it satisfies the variational minimal principle for the deformations of the class $D^{N}$, i.e. when $L^{\prime}(0)=0$, or still, when the length of $\beta$ is a critical point of the functional $L(t)$. A minimal curve $\beta$ is said to be stable minimal when $L^{\prime \prime}(0) \geq 0$, i.e. when any small deformation of the class $D^{N}$ increases the length.

Actually, as is well known, the above formulas essentially remain valid when considering any compactly supported variation of $\mathrm{D}^{N}$ also for non-closed space curves. Then (2.8) and (2.10) yield the classical result that the straight lines $\left(L^{\prime}(0)=0\right.$ for all functions $f$, implying of course $k \equiv 0$ ) are the only minimal curves in $E^{3}$, and they are moreover stable (since, always, $L^{\prime \prime}(0) \geq 0$ ). We recalled the above formulas however specifically for the closed curves in $E^{3}$, since we next want to consider various subclasses of the set $\mathrm{D}^{N}$ as determined by the behaviour of the spectral decomposition of the variational functions $f$.

\section{Curves of finite Chen type and classes $\mathbf{F}_{k}$ of functions}

The Laplace operator $\Delta$ of $S^{1}(r)$ being given by

$$
\Delta=-\frac{d^{2}}{d s^{2}}
$$

its eigenvalues are

$$
\lambda_{n}=\left(\frac{n}{r}\right)^{2} \quad, \quad(n=0,1,2, \ldots),
$$

and the corresponding eigenspaces are

$$
V_{n}=\operatorname{span}\left\{\cos \frac{n s}{r}, \sin \frac{n s}{r}\right\} \quad, \quad(n=0,1,2, \ldots) .
$$


These eigenspaces are mutually orthogonal with respect to the inner product

$$
\langle f, g\rangle:=\int_{0}^{2 \pi r} f(s) g(s) d s, \quad\left(f, g \in C^{\infty} \beta\right)
$$

and their sum $\sum_{k=0}^{\infty} V_{k}$ is dense in $C^{\infty} \beta$.

Hence the spectral decomposition of $\beta$ with respect to its Laplacian,

$$
\beta(s)=\beta_{0}+\sum_{n=1}^{\infty}\left(\bar{\beta}_{n} \cos \frac{n s}{r}+\tilde{\beta}_{n} \sin \frac{n s}{r}\right),
$$

where $\beta_{0}, \bar{\beta}_{n}$ and $\tilde{\beta}_{n}$ are certain fixed vectors in $E^{3}, \beta_{0}$ actually being the center of mass of $\beta$, is nothing but the Fourier series of $\beta$ with respect to the arclength parameter $s$. As a particular case of a general notion of (compact as well as non-compact) submanifolds of finite Chen type [2], we have the following.

Definition. A closed curve $\beta$ in $E^{3}$ is said to be of finite Chen type, FT, if its spectral decomposition (3.4) is finite; otherwise $\beta$ is said to be of infinite Chen type, $\infty T$. A finite type curve $\beta$ is said to be of $k$-type, $k T$, when the sum $\sum$ in (3.4) contains exactly $k$ non-zero terms

$\left(\bar{\beta}_{n} \cos \frac{n s}{r}+\tilde{\beta}_{n} \sin \frac{n s}{r}\right)$ each corresponding to a different eigenvalue $\lambda_{n}$. For a finite type curve, the number of the greatest eigenvalue corresponding to which a non-zero term occurs in (3.4) is called the upper order of $\beta:$ u. o. $(\beta)$.

For more information on curves of FT, see e.g. [2], [3], [4]. In particular, the circles are the only closed $1 \mathrm{~T}$ curves, all $2 \mathrm{~T}$ curves in $E^{n}$ are classified by B.Y.Chen, F.Dillen and one of the authors [4], and also all $3 \mathrm{~T}$ curves in $E^{3}$ are known through some work of L.Vrancken and both authors [8], [9] and a recent paper of D. Blair [1]. We will come back to some $2 \mathrm{~T}$ and $3 \mathrm{~T}$ closed space curves in the last section.

Similarly, every function $f \in C^{\infty} \beta$ can be decomposed in its spectral series with respect to $\Delta$, or equivalently in its Fourier series with respect to $s$ :

$$
f(s)=f_{0}+\sum_{n=1}^{\infty} f_{n}(s)
$$


whereby $f_{0}$ is a constant and $f_{n}$ is an eigenfunction belonging to $\lambda_{n}$, for $n=1,2, \ldots$, i.e.

$$
f_{n}(s)=a_{n} \cos \frac{n s}{r}+b_{n} \sin \frac{n s}{r},
$$

where $a_{n}, b_{n}$ are constants.

Definition. A function $f \in C^{\infty} \beta$ is said to be of finite Chen type, $F T$, when the sum $\sum$ in (3.5) is finite, and, in particular, $f$ is said to be of $k$-type, $k T$, when the sum $\sum$ in (3.5) contains exactly $k$ non-zero functions $f_{n}$. The number of the "highest" non-zero term appearing in the sum $\sum$ in (3.5) for a finite type function $f$ is called the upper order of $f:$ u.o. $(f)$.

Definition. A function $f \in C^{\infty} \beta$ belongs to the class $F_{k} \subset C^{\infty} \beta$ when all terms $f_{0}, f_{1}, \ldots, f_{k-1}$ in its Fourier series (3.5) are identically zero, i.e. if

$$
f \in \sum_{n=k}^{\infty} V_{n} .
$$

Clearly we have the following inclusions:

$$
C^{\infty} \beta=\mathrm{F}_{0} \supset \mathrm{F}_{1} \supset \mathrm{F}_{2} \supset \cdots \supset \mathrm{F}_{k} \supset \cdots .
$$

\section{Principal normal $k$-deformations}

Definition. A principal normal deformation $\beta \rightarrow \beta_{t}$ of a closed curve $\beta$ in $E^{3}$ is said to belong to the class $D_{k}^{N}$ of the principal normal $k$-deformations, when the function $f$ which determines the normal variational field $f N$ belongs to the set $F_{k}$.

Clearly, we have the following inclusions:

$$
\mathrm{D}^{N}=\mathrm{D}_{0}^{N} \supset \mathrm{D}_{1}^{N} \supset \mathrm{D}_{2}^{N} \supset \cdots \supset \mathrm{D}_{k}^{N} \supset \cdots .
$$

Definition. A closed curve $\beta$ in $E^{3}$ is said to be $k$-minimal, $k \in N$, if $L^{\prime}(0)=0$ for all principal normal $k$-deformations of $\beta$, or, in other 
words, if it satisfies the variational minimal principle in the class $D_{k}^{N}$. And a $k$-minimal closed curve $\beta$ in $E^{3}$ is said to be stable $k$-minimal, if $L^{\prime \prime}(0) \geq 0$ for all principal normal $k$-deformations of $\beta$.

From formula (2.10) we have the following.

Proof. Every closed $k$-minimal curve in $E^{3}$ is stable $k$-minimal.

Remark. From the previous definition and (4.1), we see that every $k$-minimal curve is automatically also $\ell$-minimal for $\ell \geq k$.

From the previous definition combined with formula (2.8), we have that $\beta$ is $k-$ minimal if and only if

$$
\int_{0}^{2 \pi r} k(s) f(s) d s=0 \quad\left(\forall f \in \mathrm{F}_{k}\right) .
$$

Since the eigenspaces $V_{n}$ of $\Delta$ are mutually orthogonal, we derive from (4.2) the following characterization of $k$-minimality.

Teorema : Let $\beta$ be a closed curve in $E^{3}$ of length $L=2 \pi r$, with arclength $s \in[0,2 \pi r]$ and curvature function $k$. Then the following statements are equivalent:

(i) $\beta$ is $k$-minimal;

(ii) $k \in \sum_{i=0}^{k-1} V_{i}$;

(iii) $k$ is a $F T$ function with $(k)<k$;

(iv) $k(s)=k_{0}+\sum_{n=1}^{k-1}\left(\bar{k}_{n} \cos \frac{n s}{r}+\widetilde{k}_{n} \sin \frac{n s}{r}\right), \quad\left(k_{0}, \bar{k}_{n}, \widetilde{k}_{n} \in R\right)$;

(v) There exists a polynomial $P$ of one variable such that $P(\Delta) k=0$.

Corolario 3 : There are no closed 0-minimal curves in $E^{3}$.

Corolario 4 : The only closed 1 -minimal curves in $E^{3}$ have constant curvature $k$. 
Corolario 5 : The closed 2-minimal curves in $E^{3}$ are the closed curves $\beta$ of length $L=2 \pi r$ in $E^{3}$ for which $k(s)=\ell+a \cos \frac{s}{r}+b \sin \frac{s}{r}$, where $\ell, a, b \in R$ and $s \in[0,2 \pi r]$ is the arclength parameter.

\section{Some examples}

In this section we discuss some non-trivial examples of $k$-minimal closed space curves, $k \geq 2$, which on one hand are known for quite some time now within the theory of submanifolds of finite Chen type, and on the other hand turned up very recently in the work of P.D.Scofield [10] on Euclidean space curves of constant precession.

Euclidean space curves of constant precession are defined by the property that, being transformed with unit speed, their centrode revolves about a fixed axis with constant angle and constant speed [10]. Here by centrode is meant, for a curve $\beta(s)$ where sis an arclength parameter, the vector $C(s)=\tau(s) T(s)+k(s) B(s)$, which at every point $\beta(s)$ of the curve determines the axis of instantaneous rotation

of the Frenet frame $\{T(s), N(s), B(s)\}$ when moving along the curve.

The following characterization of such curves is originally due to P.D.Scofield, but we will present its derivation in a somewhat different way here.

For a space curve of constant precession, it is required that $C(s)$ moves with constant speed on a circle centered at the axis of precession (a fixed line) $\ell$ in a plane perpendicular to this axis. So, to start with, we must have

$$
\|C(s)\|^{2}=k^{2}+\tau^{2}=\text { constant }
$$

say

$$
k^{2}+\tau^{2}=w^{2}, \quad\left(w \in R_{0}^{+}\right)
$$

Hence it follows that

$$
k k^{\prime}+\tau \tau^{\prime}=0
$$

such that

$$
k^{\prime}=f \tau \quad, \quad \tau^{\prime}=-f k,
$$

for some function $f$ of $s$. Next, we express that the curve $C(s)$ has constant speed: since, using the Frenet formulas,

$$
C^{\prime}(s)=\tau^{\prime} T+k^{\prime} B
$$


we must have

$$
k^{\prime 2}+\tau^{\prime 2}=\text { constant }
$$

Inserting (5.3) in (5.5), and using (5.1), we conclude that also f must be constant, say

$$
k^{\prime}=\mu \tau \quad, \quad \tau^{\prime}=-\mu k, \quad\left(\mu \in R_{0}\right),
$$

( $\mu=0$ reducing the curve $C(s)$ to a single point). Now, for $C(s)$ to describe a circle $S_{0}^{2}(\omega)$, we would need to have a fixed point $A \neq 0$, spanning the axis $\ell$, and such that $\forall s: d(A, C(s))=$ constant, say $r$, (where $\mathrm{d}$ is the Euclidean distance function), meaning then that the curve $C(s)$ lies on the intersection of two spheres $S_{0}^{2}(\omega)$ and $S_{A}^{2}(r)$, thus expressing that the angle $\theta(s)$ between the vectors $C(s)$ and the fixed vector $A$ is constant. With this in mind, for any point $\beta(s)$ on the original curve, consider a vector

$$
V(s)=\alpha(s) T(s)+\delta(s) N(s)+\gamma(s) B(s) .
$$

In order to satisfy the property we aim for, we express that the velocity vector $C^{\prime}(s)$ of $C(s)$ should be perpendicular to $V(s)$ for all $s$, yielding

$$
\alpha \tau^{\prime}+\gamma k^{\prime}=0
$$

Combining with (5.6) we then obtain that

$$
\alpha k-\gamma \tau=0
$$

such that

$$
\alpha=g \tau \quad, \quad \gamma=g k,
$$

for some function $g$ of $s$. Thus $V(s)$ should be of the form

$$
V(s)=g(\tau T+k B)+\delta N=g C+\delta N .
$$

Finally, we express that the vector $V(s)$ should be the same for all s, i.e.

$$
V^{\prime}(s)=0,
$$

and then, of course, also

$$
\|V(s)\|^{2}=\text { constant. }
$$


From (5.12) we obtain that

$$
\left(g^{\prime} \tau+g \tau^{\prime}-k \delta\right) T+\delta^{\prime} N+\left(g^{\prime} k+g k^{\prime}+\tau \delta\right) B=0,
$$

i.e. that

$$
g^{\prime} \tau+g \tau^{\prime}-k \delta=0, \quad \delta^{\prime}=0, \quad g^{\prime} k+g k^{\prime}+\tau \delta=0,
$$

so that, in particular, $\delta$ is constant, and from (5.13) we obtain that

$$
g^{2}\left(k^{2}+\tau^{2}\right)+\delta^{2}=\text { constant }
$$

which by (5.1) and the fact that we already know that $\delta$ is constant, implies that also $g$ is constant. Thus $g=C_{1}$ and

$\delta=C_{2}$ are constants, which, by (5.15), are related by

$$
C_{1} \tau^{\prime}=C_{2} k \quad, \quad C_{1} k^{\prime}=-C_{2} \tau
$$

or still, making use of (5.6), by

$$
C_{2}=-\mu C_{1}
$$

Hence

$$
V(s)=C_{1}(C(s)-\mu N(s))
$$

is a fixed vector different from 0 , (determining the axis $\ell$ ). Since any constant multiple of $V(s)$ can serve in the same purpose, we will consider in the following, in particular, the fixed vector

$$
A=C(s)-\mu N(s) \text {. }
$$

It is clear at once that

$$
d(A, C(s))=\|A-C(s)\|=\|\mu N(s)\|=|\mu|=\text { constant }
$$

say $|\mu|=r$.

From (5.6) we see that the curvature $k(s)$ and torsion $\tau(s)$ of a curve of constant precession satisfy both the differential equation

$$
F^{\prime \prime}(s)+\mu^{2} F(s)=0,
$$


which, again making use of (5.6), and eventually up to a reflection and a translation of the arclength parameter, give the following "natural equations" of such curves:

$$
k(s)= \pm \omega \sin \mu s \quad, \quad \tau(s)=\omega \cos \mu s .
$$

Teorema : ([10]). The Euclidean space curves $\beta(s)$ of constant precession are characterized by their natural equations (5.23), where $w \in R_{0}^{+}$and $\mu \in R$ are arbitrary constants.

We already know that $\|C(s)\|=\omega$, and further we will denote

$$
\|A\|=\alpha
$$

thus having

$$
\alpha=\sqrt{w^{2}+\mu^{2}} .
$$

Next, P.D.Scofield observed that since $T^{\prime}=k N$ and denoting by $\phi(s)$ the angle between $N(s)$ and the fixed vector $A$, since

$$
N(s) \cdot A=\alpha \cos \phi(s)=N(s) \cdot(C(s)-\mu N(s))=-\mu
$$

$\alpha$ and $\mu$ being constants, that also $\phi(s)$ is constant, and thus, that the spherical image $T(s)$ of a curve of constant precession $\beta(s)$ is a helix on $S_{0}^{2}(1)$, (its tangent line $N(s)$ making a constant angle with the fixed direction $\ell$ ).

Based on this, he obtains the following.

Teorema B ([10]).An arclength parametrization of a Euclidean space curve of constant precession with natural equations

$$
k(s)=-\omega \sin \mu s \quad, \quad \tau(s)=\omega \cos \mu s,
$$

is given by

$$
x(s)=\frac{\alpha+\mu}{2 \alpha(\alpha-\mu)} \sin (\alpha-\mu) s-\frac{\alpha-\mu}{2 \alpha(\alpha+\mu)} \sin (\alpha+\mu) s, y(s)=
$$




$$
\begin{array}{r}
-\frac{\alpha+\mu}{2 \alpha(\alpha-\mu)} \cos (\alpha-\mu) s+\frac{\alpha-\mu}{2 \alpha(\alpha+\mu)} \cos (\alpha+\mu) s, \\
z(s)=\frac{\omega}{\mu \alpha} \sin \mu s,
\end{array}
$$

where $w \in R_{0}^{+}, \mu \in R_{0}$ are constants and $\alpha=\sqrt{w^{2}+\mu^{2}}$. Moreover, the curve lies on the one-sheeted hyperboloid of revolution

$$
x^{2}+y^{2}-\frac{\mu^{2}}{\omega^{2}} z^{2}=\frac{4 \mu^{2}}{\omega^{4}} .
$$

And the curve is closed if and only if $\frac{\mu}{\alpha}$ is rational.

These curves are, cf $\S 3$, clearly all $3 T$-curves, except when $\alpha-\mu=$ $\mu$, i.e. $\alpha=2 \mu$, or still, using (5.25) when

$$
w=\sqrt{3}|\mu|,
$$

in which case they are 2 -curves. From Theorem B and Theorem 2, we thus have the following.

Corolario : All $2 T$-curves in $E^{3}$ of constant precession and all $3 T$-curves in $E^{3}$ of constant precession for which $\frac{\mu}{\alpha}$ is rational, are $k$-minimal curves in $E^{3}$, for some $k \geq 2$.

Remark 1. Actually, it follows from the above considerations, that the curves $\beta(s)$ of constant precession in $E^{3}$ are characterized also by the fact that their centrode $C(s)$ is a curve of constant speed lying on the sphere centered at the origin 0 of $E^{3}$.

Remark 2. Also, for curves of constant precession, it is clear from the above that $N$ revolves also with constant (complementary) angle and constant speed around the axis $\ell$. In particular this implies that the spherical image of a given curve $\beta(s)$ of constant precession, $\sigma(s)=$ $T(s)$, since $\sigma^{\prime}(s)=T^{\prime}(s)=\mathrm{k}(s) N(s)$, is a spherical helix (on $S_{0}^{2}(1)$ ).

The parametric equations of such helices are known, and so, a parametrization of the curve $\beta(s)$ of constant precession can be obtained 
by simple integration. Whereas in general, for space curves to find the parametric equations of a curve $\beta(s)$ from given natural equations is a rather complicated matter, (see e.g. Struik, Eisenhart).

\section{Some comments}

1. The above notes on $k$-minimality of space curves can trivially be extended to the study of the $k$-minimal closed curves in arbitrary dimensional Euclidean spaces $E^{m}, m>3$.

2. More generally, the theory of the $k-$ minimal compact hypersurfaces $M^{n}$ in $E^{n+1}$, or for that matter, of the $k$-minimal compact hypersurfaces $M^{n}$ in any Riemannian manifold $N^{n+1}$, could similarly be extended to a theory of $k$-minimal compact submanifolds $M^{n}$ in Riemannian manifolds $N^{m}$, with arbitrary dimensions $n$ and arbitrary codimensions $m-n$, by considering the class of $\vec{H}$-normal $k$-deformations, i.e. the deformations in the direction of the mean curvature vector field $\vec{H}$ of $M^{n}$ in $N^{m}$, for which the variational function $f \in \mathrm{F}_{k}$, i.e. $f \in \sum_{n=k}^{\infty} V_{n}$ where $V_{n}$ is the eigenspace of the Laplace operator $\Delta$ of $M^{n}$ corresponding to the eigenvalue $\lambda_{n}\left(0=\lambda_{0}<\lambda_{1}<\lambda_{2}<\ldots<+\infty\right)$. 


\section{References}

[1] D. E. Blair, Classification of 3-type curves. Soochow J.Math., 21, pp. 145-158, (1995).

[2] B. Y. Chen, Total mean curvature and submanifolds of finite type. World Scientific. Singapore, (1984).

[3] B. Y.Chen, J.Deprez, F.Dillen, L.Verstraelen and L.Vrancken, Curves of finite type. Geometry and Topology of Submanifolds, II, World Scientific. Singapore, pp. 76-110, (1990).

[4] B. Y. Chen, F.Dillen and L.Verstraelen, Finite type space curves. Soochow J.Math., 12, pp. 1-10, (1986).

[5] B. Y. Chen, F. Dillen, L. Verstraelen and L. Vrancken, A variational minimal principle characterizes submanifolds of finite type CR Acad. Sc. Paris 317, pp. 961-965, (1993).

[6] B. Y. Chen, F. Dillen, L. Verstraelen and L. Vrancken, Compact hypersurfaces determined by a spectral variational principle Kyushu Journal of Math. 49, pp. 103-121, (1995).

[7] J. Deprez, F. Dillen and L. Vrancken, Finite type curves on quadrics Chinese J. Math., 18, pp. 95-121, (1990).

[8] M. Petrović, L. Verstraelen and L. Vrancken, 3-type curves on ellipsiods of revolution Preprint series, Dept. Math. Katholieke Univ. Leuven, 2, pp. 31-49, (1990).

[9] M. Petrović, L. Verstraelen and L. Vrancken, 3-type curves on hyperboloids of revolution and on cones of revolution Publ. Inst. Math. Belgrade, 59 (73), pp. 138-152, (1996).

[10] P. D. Scofield, Curves of constant precession Amer. Math. Monthly 102 (6), pp. 531-537, (1995). 
Received : June, 1999.

\section{Petrović}

University of Kragujevac

Faculty of Science

Institute of Mathematics

Radoja Domanovića 12

34000 Kragujevac

Yugoslavia

J. Verstraelen and L. Verstraelen

Katholieke Universiteit Brussel

Group of Exact Sciences

Vrijheidslaan 17

B-1080 Brussel

Belgium

\section{J. Verstraelen}

Economische Hogeschool Sint-Aloysius

Stormstraat 2

B-1000 Brussel

Belgium

and

\section{Verstraelen}

Katholieke Universiteit Leuven

Departement Wiskunde

Celestijnenlaan 200 B

B-3001 Heverlee (Leuven)

Belgium 